\title{
乌s \\ Phase combination for self-cancellation of magnetic force in undulators
}

\author{
Ryota Kinjo* and Takashi Tanaka \\ RIKEN SPring-8 Center, 1-1-1, Kouto, Sayo-cho, Sayo-gun, Hyogo, Japan
}

(Received 14 October 2014; published 23 December 2014)

\begin{abstract}
A simple arrangement of undulator magnets having nearly zero magnetic force between the upper and lower magnet arrays is proposed, which significantly simplifies the mechanical structure of undulators. In this arrangement, each magnet array is divided into a number of sections, half of which are phase-shifted according to a particular rule without breaking the periodic condition required for the undulator field. Calculation results show that, when the gap over period is in practical range, more than or equal to $1 / 18$, the magnetic force can be reduced down to less than $1 / 50$ of that in the Halbach undulator by applying the proposed scheme.
\end{abstract}

DOI: 10.1103/PhysRevSTAB.17.122401

PACS numbers: 07.85.Qe, 41.60.Cr

\section{INTRODUCTION}

Low-emittance storage rings under construction and planning, as well as the third-generation synchrotron radiation (SR) light sources, require a great number of undulators. X-ray free electron lasers (XFELs) with the self-amplified spontaneous emission (SASE) scheme require a long undulator section, typically with the length from several tens to hundreds of meters [1,2]. Moreover, recently developed techniques to enhance the temporal coherence of SASE $[3,4]$ and a scheme to generate an attosecond XFEL pulse [5] assume a further long undulator, because laser saturation lengths in these schemes are longer than that of the original SASE. Reduction of the cost of undulators, especially of variable-gap undulators, which have been and will be installed in the future SR and XFEL facilities [6], is thus strongly demanded.

Variable-gap undulators usually require rigid mechanical components and frames to control the magnet gap precisely against the large attractive force. Moreover, if the undulator is of the in-vacuum type, a large number of components are necessary to distribute the mechanical load along the undulator axis and to avoid deformation of the magnetic arrays that are located inside the vacuum chamber. Such a conventional undulator design gives rise to structural issues that most of the weight, dimensions, and cost of the undulator are attributable to the auxiliary apparatus but not to the core part, i.e., the magnet arrays.

The above discussion in turn gives us a new concept of undulator design; if the attractive force is canceled out, the heavy and large mechanical frame is no longer required, and then the undulator can be much more lightweight and

\footnotetext{
r-kinjo@spring8.or.jp

Published by the American Physical Society under the terms of the Creative Commons Attribution 3.0 License. Further distribution of this work must maintain attribution to the author(s) and the published article's title, journal citation, and DOI.
}

compact. As a result, the cost and lead time of construction, transportation, and installation are significantly reduced. Up to now, two different methods have been developed to cancel the attractive force. One is the mechanical system composed of a number of springs having different lengths and spring constants attached to the both sides of the main magnets, which was applied to an in-vacuum wiggler developed at Synchrotron SOLEIL [7]. The other is the magnetic system composed of two rows of magnet arrays generating a repulsive force attached to both sides of the main magnets, which was applied to the in-vacuum revolver undulator developed at SPring-8 [8]. Although both systems worked well for their own purposes, they have difficulties in application to the new undulator concept. In the former method, the precise magnetic measurement indispensable for undulator field correction is not possible with the conventional instrument, and the gap dependency of the repulsive force generated by the springs is somewhat different from that of the attractive force. In the latter method, the number of magnets and magnet holders is 3 times as large as that of the main magnet array, which increases the cost, time, and effort to manufacture the magnet arrays. Recently, a new undulator concept based on a cost-effective force cancellation system has been proposed at SPring-8 [9], which takes advantage of multipole monolithic magnets $[10,11]$. Design of the lightweight, compact, and cost-effective undulator based on the force cancellation system is under progress.

In this paper, we propose another solution for the lightweight, compact, and cost-effective undulator, which is hereinafter referred to as the phase-combined undulator (PCU). This undulator intrinsically has no net magnetic force without any force cancellation system. In this arrangement, each magnet array is divided into a number of sections, half of which are phase-shifted (longitudinally displaced) according to a particular rule without breaking the periodic condition required for the undulator field. First, the principle of the PCU is described, and the analytical and 
numerical calculation methods for the magnetic field and force are introduced. Next, the results of the calculations performed to examine the performance of the proposed concept are described. Finally, those results are discussed.

\section{THEORY}

\section{A. Magnetic field and force in a phase-shifted configuration}

The concept of the PCU is based on the fact that, by shifting the relative phase between the upper and lower Halbach arrays, the magnetic force in between changes. This is known in the context of the polarization switchover in helical undulators, for example, the APPLE-II undulator in which four Halbach arrays are placed at a certain phase.

Let us first recall the analytical formula to give the magnetic distribution of the Halbach undulator (HU). For this purpose, we consider an undulator infinitely long and wide and consisting of magnets having the magnetic susceptibility of zero. Under such an assumption, we can treat the magnetic field and force in the undulator perfectly by using the current sheet equivalent model in which the magnet having the remanent field $B_{r}$ is replaced by the surface current having a density of $J=B_{r} / \mu_{0}[\mathrm{~A} / \mathrm{m}]$. Then in the complex plane spanned by two coordinates $z$ and $y$, where $z$ is the longitudinal direction along the electron beam path and $y$ is perpendicular to $z$, we can define the complex magnetic field $B^{*}(\xi=z+\mathrm{i} y)=B_{z}(\xi)-\mathrm{i} B_{y}(\xi)$ and the complex magnetic force on the upper magnet array $F_{\mathrm{UL}}^{*}=F_{z}-\mathrm{i} F_{y}$. The magnetic fields $B_{L}^{*}(\xi)$ and $B_{U}^{*}(\xi)$ generated by the lower and upper magnets, respectively, are [12]

$$
\begin{aligned}
& B_{\mathrm{L}}^{*}(\xi)=-\frac{\mathrm{i}}{2} \sum_{n=1+\nu M}^{\infty} B_{n, 0} e^{\mathrm{i} n k_{u} z} e^{-n k_{u} y} \quad\left(y>-\frac{g}{2}\right), \\
& B_{\mathrm{U}}^{*}(\xi)=-\frac{\mathrm{i}}{2} \sum_{n=1+\nu M}^{\infty} B_{n, 0} e^{-\mathrm{i} n k_{u} z} e^{n k_{u} y} \quad\left(y<+\frac{g}{2}\right),
\end{aligned}
$$

with

$$
B_{n, 0}=2 B_{r} \operatorname{sinc}(n \pi / M) e^{-n k_{u} g / 2}\left(1-e^{-n k_{u} h}\right),
$$

where $n=1+\nu M(\nu \in \mathbb{Z})$ is the harmonic number of the magnetic field, $M$ is the number of magnets per period, $B_{n, 0}$ is the amplitude of the $n$th harmonic, $k_{u}$ is the wave number which is defined by using the undulator period $\lambda_{u}$ as $k_{u}=2 \pi / \lambda_{u}, g$ is the gap between the upper and lower magnet arrays, and $h$ is the height of the magnet. Note that $M=4$ is selected in most cases for practical reasons, which is also the main subject of this paper. Summing $B_{L}^{*}$ and $B_{U}^{*}$, one obtains the well-known formula for HU.

The deflection parameter $K$ ( $K$ value) which determines the wavelength of undulator radiation is given by [13]

$$
K^{2}=2\left\langle[l(z)-\langle l(z)\rangle]^{2}\right\rangle,
$$

with

$$
l(z)=\frac{e}{m_{e} c} \int^{z} B_{y}\left(z^{\prime}\right) d z^{\prime}
$$

where $\langle\ldots\rangle$ means the average over the length $\lambda_{u}, e$ and $m_{e}$ are the charge and mass of an electron, respectively, and $c$ is the speed of light. Therefore, the $K$ value for HU $K_{0}$ is

$$
K_{0}=\frac{e \lambda_{u}}{2 \pi m_{e} c} \sqrt{\sum_{n=1+\nu M}^{\infty}\left(\frac{B_{n, 0}}{n}\right)^{2}} .
$$

Next let us calculate the magnetic force between the upper and lower magnet arrays. By integrating the force per unit surface $\mathbf{f}=\mathbf{J} \times \mathbf{B}\left(f^{*}=\mathrm{i} B_{r} B^{*} / \mu_{0}\right)$ along the current sheet in the upper volume, we obtain the force acting on the upper $4 N$ magnets having the finite width $W$ in $N$ periods:

$$
F_{\mathrm{UL}}^{*}=\mathrm{i} F_{0},
$$

with

$$
\begin{aligned}
& F_{0}=\sum_{n=1+\nu M}^{\infty} F_{n}, \\
& F_{n}=\frac{N \lambda_{u} W}{4 \mu_{0}} B_{n, 0}^{2} .
\end{aligned}
$$

The $y$ component of $F_{\mathrm{UL}}^{*}$ has a minus sign representing that the upper magnet array is attracted by the lower magnet array.

Let us briefly check the dependence of the magnetic field and force on the relative phase shift (longitudinal displacement) between the lower and upper magnet arrays when only the upper magnet array is displaced by $\Delta_{\mathrm{UL}}$ as shown in Figs. 1(a)-1(d). The pair of small arrows indicates the attractive (blue) or repulsive (red) force between two magnets. The total forces acting on the magnet beams are (a) attractive, (b) negative shear, (c) repulsive, and (d) positive shear forces as drawn by the large arrows (white), where the directions of the shear forces are defined in the following. To be more specific, the magnetic field on axis, the magnetic force, and the $K$ value with the relative longitudinal displacement $\Delta_{\mathrm{UL}}$ are, respectively,

$$
\begin{aligned}
B^{*}\left(z, \Delta_{\mathrm{UL}}\right)= & -\mathrm{i} \sum_{n=1+\nu M}^{\infty} B_{n, 0} \cos \\
& \times\left[n k_{u}\left(z-\Delta_{\mathrm{UL}} / 2\right)\right] e^{\mathrm{i} n k_{u} \Delta_{\mathrm{UL}} / 2}, \\
F_{\mathrm{UL}}^{*}\left(\Delta_{\mathrm{UL}}\right)= & \mathrm{i} \sum_{n=1+\nu M}^{\infty} F_{n} e^{\mathrm{i} n k_{u} \Delta_{\mathrm{UL}}},
\end{aligned}
$$



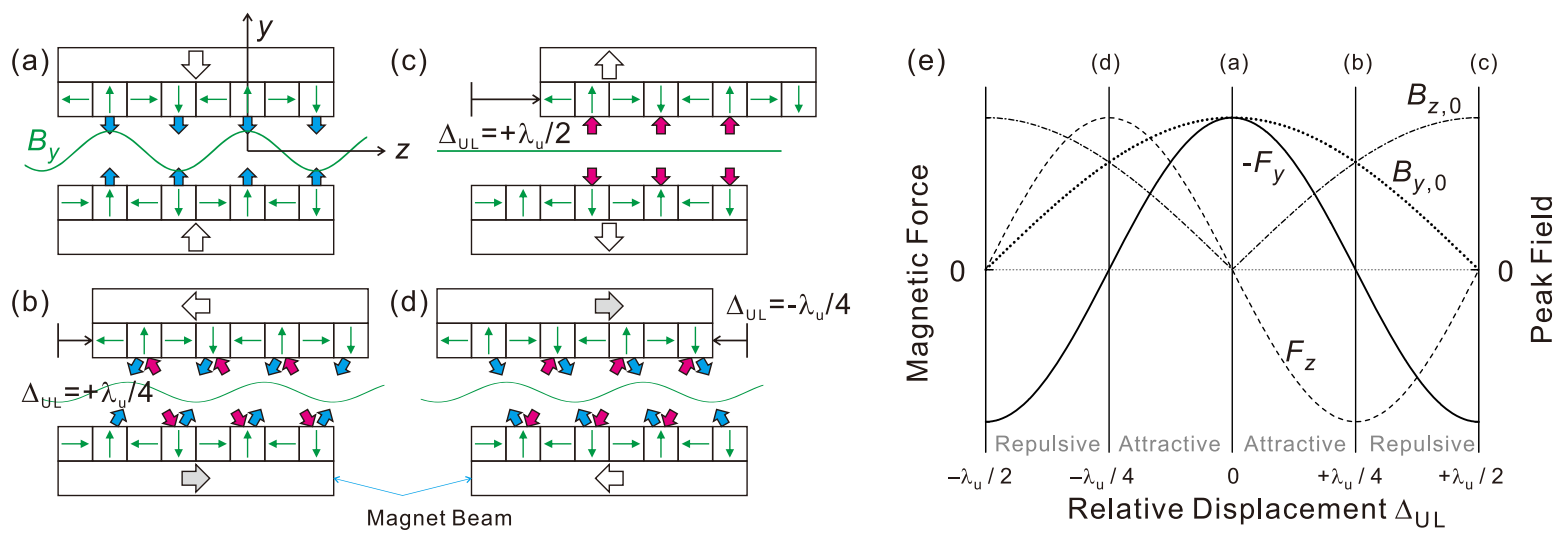

FIG. 1. Halbach arrays with the relative longitudinal displacement between the upper and lower magnet arrays $\Delta_{\mathrm{UL}}$ of (a) zero, (b) $+\lambda_{u} / 4$, (c) $+\lambda_{u} / 2$, and (d) $-\lambda_{u} / 4$. The pair of small arrows indicates the attractive (blue) or repulsive (red) force between two magnets. The total forces acting on the magnet beam are drawn by the large arrows (white). The phase diagram of the forces and the peak fields are drawn in (e).

$K\left(\Delta_{\mathrm{UL}}\right)=\frac{e \lambda_{u}}{2 \pi m_{e} c} \sqrt{\sum_{n=1+\nu M}^{\infty}\left(\frac{B_{n, 0} \cos \left(n k_{u} \Delta_{\mathrm{UL}} / 2\right)}{n}\right)^{2}}$.

The fundamental of the field $(n=1)$ has peaks at $z=\Delta_{\mathrm{UL}} / 2 \pm m \pi(m \in \mathbb{Z})$. Contributions from the higher harmonics are negligible, and thus the peak amplitudes are

$$
\begin{aligned}
& B_{z, 0}\left(\Delta_{\mathrm{UL}}\right) \simeq\left|\sin \left(k_{u} \Delta_{\mathrm{UL}} / 2\right)\right| B_{0}, \\
& B_{y, 0}\left(\Delta_{\mathrm{UL}}\right) \simeq\left|\cos \left(k_{u} \Delta_{\mathrm{UL}} / 2\right)\right| B_{0} .
\end{aligned}
$$

We have also the magnetic force:

$$
\begin{aligned}
& F_{z}\left(\Delta_{\mathrm{UL}}\right) \simeq-\sin \left(k_{u} \Delta_{\mathrm{UL}}\right) F_{0}, \\
& F_{y}\left(\Delta_{\mathrm{UL}}\right) \simeq-\cos \left(k_{u} \Delta_{\mathrm{UL}}\right) F_{0} .
\end{aligned}
$$

$F_{z}$ and $F_{y}$ indicate the magnetic forces in the longitudinal and vertical directions, respectively, acting on the upper array. Thus, the positive and negative values of $F_{y}$ indicate the repulsive and attractive force, respectively, between them. The positive and negative values of $F_{z}$ are hereinafter referred to as positive and negative shear forces, respectively. Figure 1(e) shows the dependence of the peak magnetic fields and the magnetic forces in the $y$ and $z$ directions on the phase difference between the upper and lower magnet arrays calculated by the analytical solutions derived above. We find that $F_{y}$ reduces to 0 at $\Delta_{\mathrm{UL}}=$ $\pm \lambda_{u} / 4$, which means that the attractive force can be completely eliminated, though $B_{y, 0}$ gets down to smaller than the original value. Moreover, the shear force $F_{z}$ appears, with the same strength as the original value of $F_{y}$, which means that the undulator mechanical frame should be rigid as well even without the attractive force. In the following, the method to cancel out $F_{z}$ is introduced.

\section{B. Principle of PCU}

Figure 2 shows the conceptual drawing of the PCU. The two phase-shifted configurations (a) and (b) are combined as (c) PCU with $D=2$ and (d) PCU with $D=N_{u}$, where $D$ denotes the number of sections to be explained later in more detail. The symbol $b$ indicates that the upper magnets are displaced by $\Delta_{\mathrm{UL}}=-\lambda_{u} / 4$ in the section, and the symbol $\sharp$ indicates that the lower magnets are displaced by $\Delta_{\mathrm{LU}}=-\lambda_{u} / 4$. In the PCU, the positive and negative shear forces, which appear at the nonzero phase shift, in the configuration (a) and (b) are combined to cancel out $F_{z}$ with keeping the periodicity of $B_{y}$. On the other hand, the polarity of $B_{z}$ flips every other section, which results in the periodicity with a longer period than $\lambda_{u}$. The periodicity change in $B_{z}$ obviously has little impact on the radiation properties but may have some on the electron beam dynamics, which is out of the scope of this paper.

Let us consider the case when the PCU $(D=2)$ is divided into two parts at the center $\left(z_{d}=-3 \lambda_{u} / 8\right)$ and assume that the equations still work individually in the section $b\left(z \leq z_{d}\right)$ and the section $\sharp\left(z>z_{d}\right)$. Then, the field and force equations can be expressed by

$$
\begin{aligned}
B^{*}(z)= & \begin{cases}B^{*}\left(z,-\lambda_{u} / 4\right) & \left(z \leq z_{d}\right) \\
B^{*}\left(z+\lambda_{u} / 4,+\lambda_{u} / 4\right) & \left(z>z_{d}\right)\end{cases} \\
= & -\operatorname{sgn}\left(z-z_{d}\right) B_{z}\left(z,-\lambda_{u} / 4\right) \\
& -\mathrm{i} B_{y}\left(z,-\lambda_{u} / 4\right) . \\
F_{\mathrm{UL}}^{*}= & F_{z}-\mathrm{i} F_{y}= \begin{cases}-F_{0} / 2 & (\text { Sec. b }), \\
+F_{0} / 2 & (\text { Sec. } \sharp) .\end{cases}
\end{aligned}
$$

The validity of this assumption is discussed later by comparing with the numerical results. Because the $B_{y}$ component is the same as the case when $\Delta_{\mathrm{UL}}=-\lambda_{u} / 4$ in both sections, then, by substituting it into Eq. (8), we obtain the $K$ value of the PCU: 


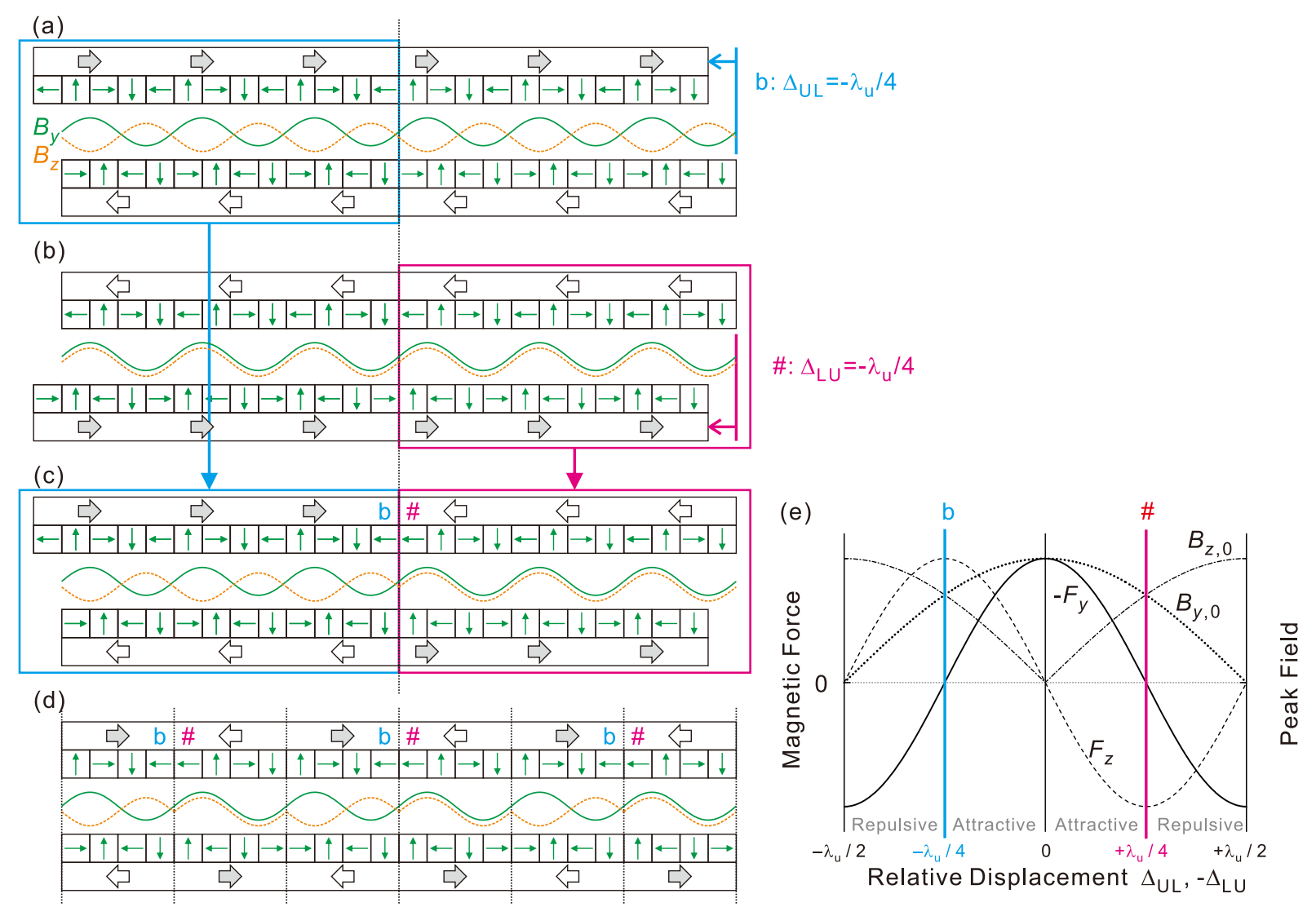

FIG. 2. Conceptual drawing of the PCU. (a) The upper and (b) the lower phase-shifted configuration are combined as (c) the PCU with $D=2$ and (d) the PCU with $D=N_{u}$. The symbol b indicates that the upper magnets are displaced by $\Delta_{\mathrm{UL}}=-\lambda_{u} / 4$ in the section, and the symbol $\sharp$ indicates that the lower magnets are displaced by $\Delta_{\mathrm{LU}}=-\lambda_{u} / 4$ as shown in (e) the phase diagram.

$$
K=\frac{1}{\sqrt{2}} K_{0} .
$$

In the same manner, the PCU configuration with $2<$ $D \leq N_{u}$ can be considered. Each section has $N / D$ periods. The result is that $B_{z}$ and $F_{z}$ change their sign for each section, and the $K$ value is identical to that in the PCU $(D=2)$.

The forces within a single section are $F_{y}^{\mathrm{s}}=0$ and $F_{z}^{\mathrm{s}}= \pm F_{0} / D$, which results in nearly zero magnetic force in total. It should be noted, however, that extra magnetic force $\left|F_{y, 0}^{\mathrm{j}}\right| \ll F_{0}$ arises at the $D-1$ joints, where the periodicity of $\lambda_{u}$ is violated. As a result, the PCU has a small but finite attractive or repulsive force $F_{y} \propto D-1$. Moreover, the tensile or compressive force $2 F_{z}^{\mathrm{s}}$ act on each joint.

\section{Fine adjustment methods for magnetic force in PCU}

There are two methods to make a fine adjustment of the magnetic force in the PCU. One is by the additional phase shift, which means that the relative longitudinal displacement of $\delta$ is added to each section as shown in Fig. 3. The symbol $b^{\prime}$ indicates that the upper magnets are displaced by $\Delta_{\mathrm{UL}}=-\lambda_{u} / 4-\delta$ in the section, and the symbol $\sharp^{\prime}$ indicates that the lower magnets are displaced by $\Delta_{\mathrm{LU}}=$ $-\lambda_{u} / 4-\delta$. The phase diagram in Fig. 3 shows that $F_{y}$ in sections $b^{\prime}$ and $\sharp^{\prime}$ change in the same direction, while $F_{z}$ in those change to the opposite directions. Then, the total $F_{y}$ in the PCU changes, while $F_{z}$ is still being balanced in total. The other is by the easy axis rotation, which means that the magnetizations of the magnets are rotated by $-\theta$ in section $b$ and $+\theta$ in section $\sharp$ as shown in Fig. 4 .

Let us consider the adjustment method by the additional phase shift. Considering the longitudinal displacements $\Delta_{\mathrm{UL}}=-\lambda_{u} / 4-\delta$ and $\Delta_{\mathrm{LU}}=-\lambda_{u} / 4-\delta$ in the same manner as Eq. (9), we obtain

$$
\begin{aligned}
B^{*}(z)= & \begin{cases}B^{*}\left(z,-\lambda_{u} / 4-\delta\right) & \left(z \leq z_{d}\right) \\
B^{*}\left(z+\lambda_{u} / 4+\delta,+\lambda_{u} / 4+\delta\right) & \left(z>z_{d}\right)\end{cases} \\
= & -\operatorname{sgn}\left(z-z_{d}\right) B_{z}\left(z,-\lambda_{u} / 4-\delta\right) \\
& -\mathrm{i} B_{y}\left(z,-\lambda_{u} / 4-\delta\right), \\
F_{\mathrm{UL}}^{*}= & \sum_{n=1+\nu M}^{\infty} F_{n} \begin{cases}\left(-\cos \left(n k_{u} \delta\right)-\mathrm{i} \sin \left(n k_{u} \delta\right)\right) & (\text { Sec. b) }) \\
\left(+\cos \left(n k_{u} \delta\right)-\mathrm{i} \sin \left(n k_{u} \delta\right)\right) & (\text { Sec. } \sharp)\end{cases} \\
\simeq & \frac{F_{0}}{2} \begin{cases}\left(-\cos \left(k_{u} \delta\right)-\mathrm{i} \sin \left(k_{u} \delta\right)\right) & (\text { Sect. b), } \\
\left(+\cos \left(k_{u} \delta\right)-\mathrm{i} \sin \left(k_{u} \delta\right)\right) & (\text { Sect. } \sharp) .\end{cases}
\end{aligned}
$$



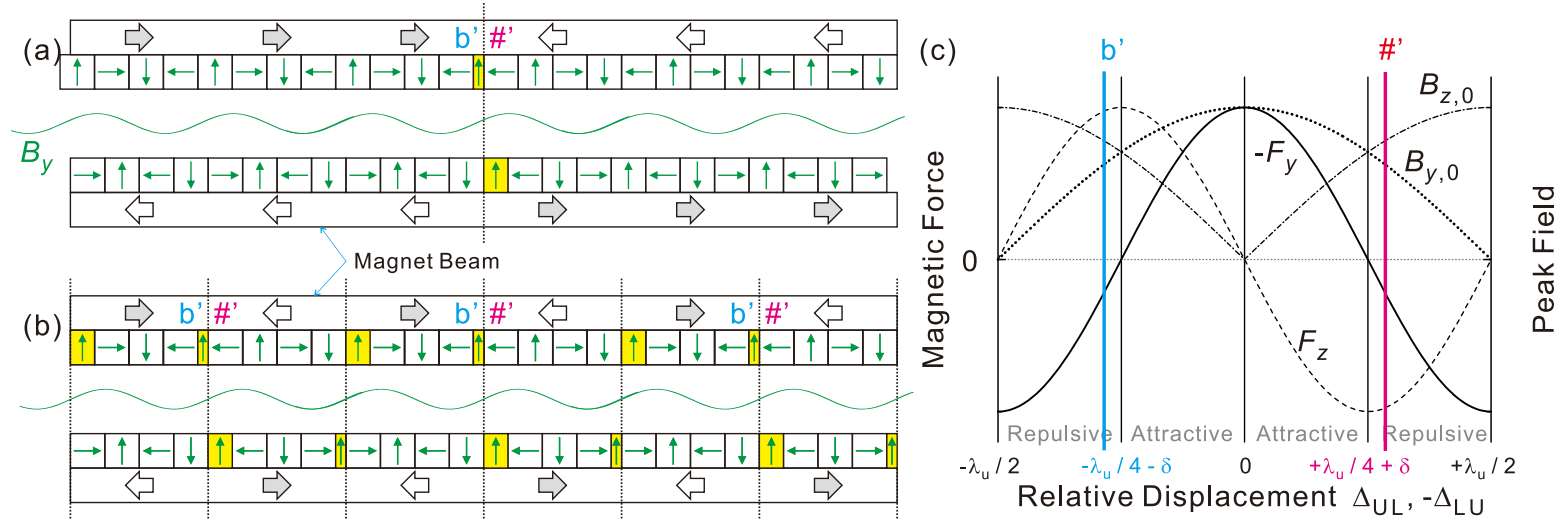

FIG. 3. Method for a fine adjustment of the magnetic force in the PCU (a) $D=2$ and (b) $D=N_{u}$ by the additional phase shift.

To make an adjustment by, for example, $F_{y}= \pm 5 \% F_{0}$, the additional longitudinal displacement is $\delta \approx \pm 0.8 \% \lambda_{u}$. The change in $K$ value is approximately $\pm 2.5 \%$.

Let us consider the adjustment method by the easy axis rotation. From Halbach's easy axis rotation theorem [14], the magnetic fields in the gap are rotated by $+\theta$ in section $b$ and $-\theta$ in section $\sharp$. Then, we obtain

$$
\begin{aligned}
B^{*}(z)= & \begin{cases}B^{*}\left(z,+\lambda_{u} / 4\right) e^{+\mathrm{i} \theta} & \left(z \leq z_{d}\right) \\
B^{*}\left(z-\lambda_{u} / 4,-\lambda_{u} / 4\right) e^{-\mathrm{i} \theta} & \left(z>z_{d}\right)\end{cases} \\
= & \sum_{n=1,5,9, \ldots}^{\infty} B_{n} \cos \left[n k_{u}\left(z-\lambda_{u} / 4\right)\right] \\
& \times\left[\operatorname{sgn}\left(z-z_{d}\right) \sin (n \pi / 4+\theta)-\mathrm{i} \cos (n \pi / 4+\theta)\right],
\end{aligned}
$$

$$
F_{\mathrm{UL}}^{*}=\frac{F_{0}}{2} \begin{cases}-\cos 2 \theta-\mathrm{i} \sin 2 \theta & (\text { Sec. b) }, \\ +\cos 2 \theta-\mathrm{i} \sin 2 \theta & (\text { Sec. } \sharp) .\end{cases}
$$

To make an adjustment by $F_{y}= \pm 5 \% F_{0}$, the magnetization rotation is $\theta \approx \pm 1.4^{\circ}$. The change in $K$ value is approximately $\pm 2.5 \%$. By comparing Eqs. (13) and (15), both methods lead to equivalent results if

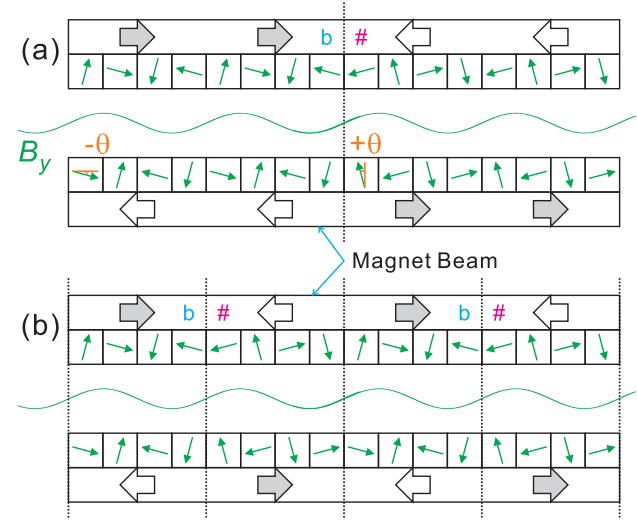

FIG. 4. Method for a fine adjustment of the magnetic force in the PCU (a) $D=2$ and (b) $D=N_{u}$ by the easy axis rotation.

$$
\theta \approx \pi \delta / \lambda_{u} \quad\left(\delta \ll \lambda_{u}\right)
$$

Because the additional phase shift equals the easy axis rotation and is much more complex for $D=N_{u}$ from a practical point of view, we treat only the easy axis rotation for $D=N_{u}$ later.

\section{EXAMPLE}

In order to examine the performance of the PCU, the magnetic fields and forces in the $\mathrm{HU}$, the PCU $(D=2)$, and the PCU $\left(D=N_{u}\right)$ with the period of $\lambda_{u}=18 \mathrm{~mm}$, the magnet width of $W=40 \mathrm{~mm}$ and the magnet height of $h=20 \mathrm{~mm}$ were calculated in both the analytical and numerical methods, in the gap range from $g=1$ to $15 \mathrm{~mm}$. The numerical calculation was performed by the threedimensional magnetostatic calculation code RADIA [15], in which the magnetic susceptibilities perpendicular and parallel to the easy axis were assumed to be 0.05 and 0.17 , respectively. The self-demagnetizing effect depending on the shape of the magnet was also taken into account.

The magnetic field distributions along the $z$ axis in the HU, the PCU $(D=2)$, and the PCU $\left(D=N_{u}\right)$ are shown in Fig. 5 at the gap of $g=1 \mathrm{~mm}$ and $g=9 \mathrm{~mm}$. The analytical and numerical results of $B_{y}$ show good agreement, while those of $B_{z}$ have some discrepancies near the joints. It should be emphasized that $B_{y}$ is periodic with the period of $\lambda_{u}$ in the whole range for both PCUs, and in practice we find no difference between $D=2$ and $D=N_{u}$, while the periodicity of $B_{z}$ depends on $D$. The $K$ values calculated by the integral of the numerical fields are $72 \%$ and $71 \%$ of that of $\mathrm{HU}$ at $g=1 \mathrm{~mm}$ and $g=9 \mathrm{~mm}$, respectively, regardless of the value of $D$, which means that $D$ can be selected just in terms of how to reduce the magnetic force. These $K$ values are consistent with that predicted in Eq. (11).

The magnetic forces in the HU, the PCU $(D=2)$, and the PCU $\left(D=N_{u}\right)$ are shown in Fig. 6 as a function of the gap. The numerically calculated magnetic forces in the PCUs are much smaller than that in HU and, however, have 


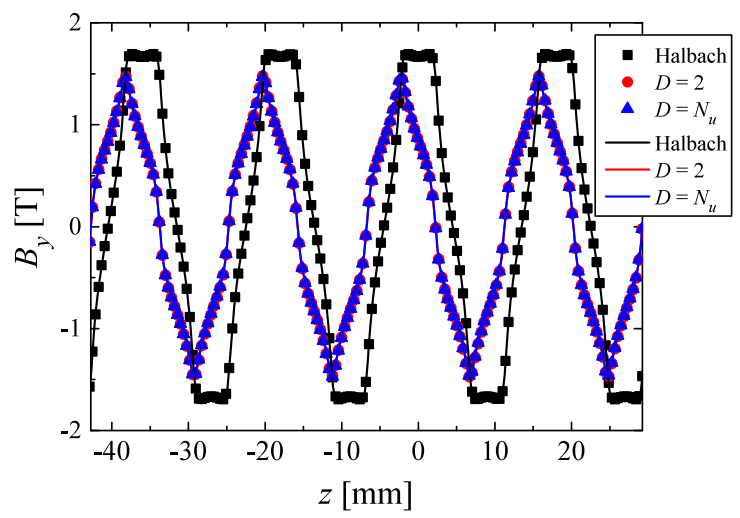

(a) $B_{y}$ at $g=1 \mathrm{~mm}$

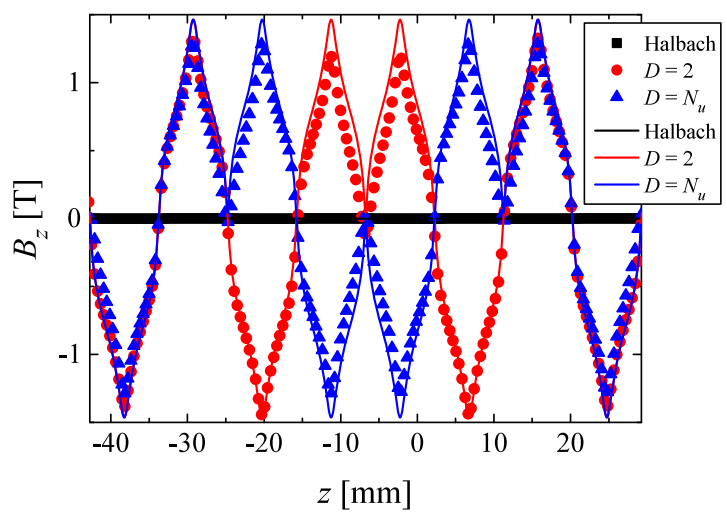

(c) $B_{z}$ at $g=1 \mathrm{~mm}$

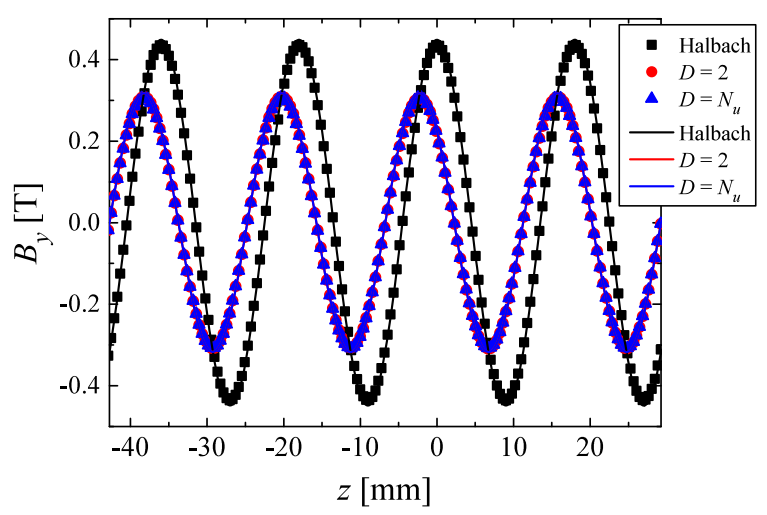

(b) $B_{y}$ at $g=9 \mathrm{~mm}$

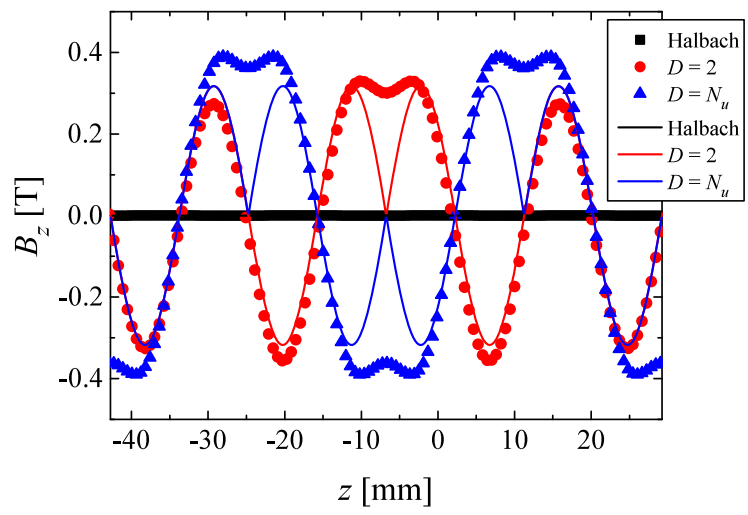

(d) $B_{z}$ at $g=9 \mathrm{~mm}$

FIG. 5. Analytically (lines) and numerically (symbols) calculated magnetic field in the HU, the PCU $(D=2)$, and the PCU $\left(D=N_{u}\right)$. The joints are at $z=-3 \lambda_{u} / 8$ for $D=2$ and $z=-3 \lambda_{u} / 8+m \lambda_{u}(m \in \mathbb{Z})$ for $D=N_{u}$.

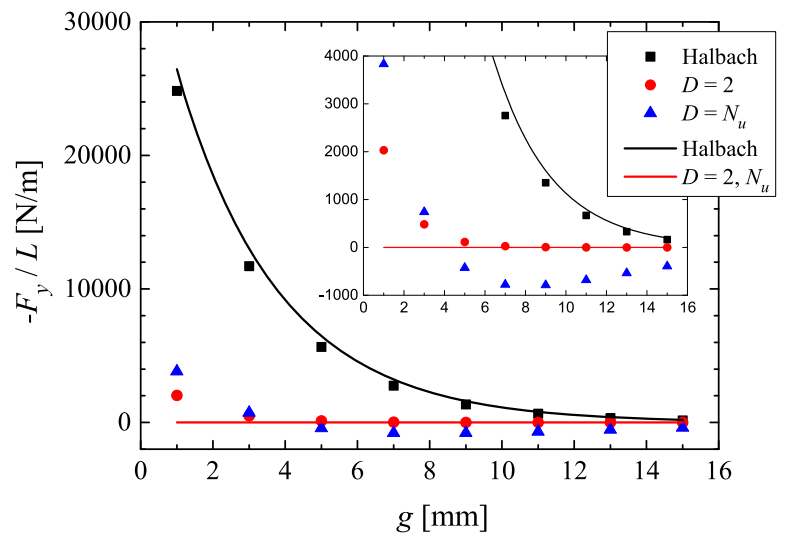

FIG. 6. Analytically (solid lines) and numerically (symbols) calculated magnetic forces for the HU, the PCU $(D=2)$, and the $\operatorname{PCU}\left(D=N_{u}\right)$.

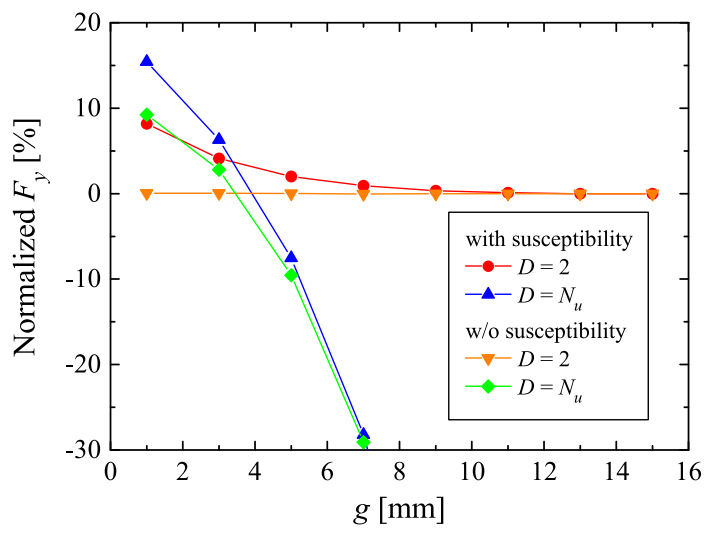

FIG. 7. Numerically calculated magnetic forces for the PCU $(D=2)$ and the PCU $\left(D=N_{u}\right)$, with and without magnetic susceptibility. The magnetic forces at the respective gap values are normalized by those for HU. 


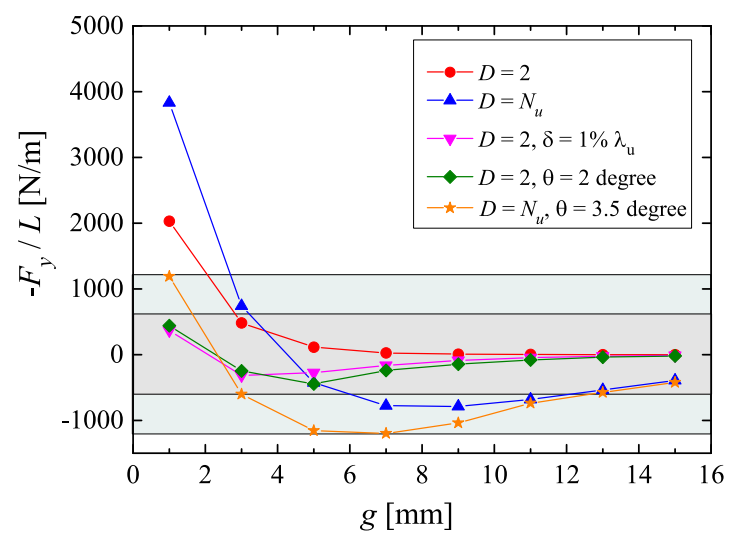

FIG. 8. Numerically calculated magnetic forces for the PCU $(D=2)$ and the PCU $\left(D=N_{u}\right)$ with and without the fine adjustments by the additional shift and the easy axis rotation.

non-negligible values, while the analytical formula indicates zero magnetic force. The forces in the PCU $(D=2)$ are smaller than those in the PCU $\left(D=N_{u}\right)$ and are attractive over the whole range of interest, while those of the PCU $\left(D=N_{u}\right)$ change sign near the gap of $g=4 \mathrm{~mm}$; the forces are attractive in the small gap and repulsive in the large gap.

To clarify the origin of the nonzero force, the magnetic forces calculated by the numerical methods with and without the magnetic susceptibility were compared, which is shown in Fig. 7. The magnetic forces at the respective gap values are normalized by those for HU. The force curves with the susceptibility approach asymptotically to the curves without it when the gap is opened. This is because the susceptibility works to strengthen the attractive force and weaken the repulsive force. We find that the forces even without the susceptibility for the PCU $\left(D=N_{u}\right)$ are not negligible, which may be due to the violation of the periodic condition.

Figure 8 shows the numerically calculated magnetic forces for the PCU $(D=2)$ and the PCU $\left(D=N_{u}\right)$ with and without the fine adjustments by the additional phase shift and the easy axis rotation. Both adjustment methods reduce the maximum force $\left|F_{y}\right| / L$ to less than $500 \mathrm{~N} / \mathrm{m}$ for $D=2$. The adjustment method by the easy axis rotation reduces the maximum force to $1200 \mathrm{~N} / \mathrm{m}$ for $D=N_{u}$.

TABLE I. Maximum absolute forces.

\begin{tabular}{lc}
\hline \hline$g_{\min } \geq 3 \mathrm{~mm}\left(g_{\min } / \lambda_{u} \geq 1 / 6\right)$ & Max. $\left|F_{y}\right| / L[\mathrm{~N} / \mathrm{m}]$ \\
\hline $\mathrm{HU}$ & 12000 \\
$\mathrm{PCU}\left(2 \leq D \leq N_{u}\right)$ & $500-800$ \\
\hline \hline$g_{\min } \geq 1 \mathrm{~mm}\left(g_{\min } / \lambda_{u} \geq 1 / 18\right)$ & Max. $\left|F_{y}\right| / L[\mathrm{~N} / \mathrm{m}]$ \\
\hline $\mathrm{HU}$ & 25000 \\
PCU $\left(2 \leq D \leq N_{u}\right)$ & $2100-3900$ \\
With adjustments & $400-1200$ \\
\hline \hline
\end{tabular}

\section{DISCUSSION}

The results described above can be summarized in Table I in terms of the maximum force per unit length when the minimum operation gap $g_{\min }$ is limited to a certain value. For example, the maximum force was $500-800 \mathrm{~N} / \mathrm{m}$ at $g_{\min }=3 \mathrm{~mm}$ and $2100-3900$ and $400-1200 \mathrm{~N} / \mathrm{m}$ at $g_{\min }=1 \mathrm{~mm}$ with and without the fine adjustments, respectively. It should be noted that these results are universal as long as the gap-to-period ratio is kept constant. Here, we assume that the magnet height is large enough that $h \gtrsim \lambda_{u}$; then, $1-e^{-n k_{u} h} \sim 1$. From a practical point of view, the magnetic force does not need to be completely zero. For example, let us consider the total weight of the magnets, magnet holders, and magnet beam in the half side, which have to be supported by the outer mechanics. In practice, the total weight of these components is about $60 \mathrm{~kg} / \mathrm{m}$ in the half side. If $g_{\min } / \lambda_{u}$ is greater than or equal to $1 / 6$, any adjustment is not required, because the magnetic force is less than or comparable to the gravity. If $g_{\min }$ is much smaller than that, the adjustment can be an option. With the adjustment methods, the maximum force for $D=2$ even at $g_{\min } / \lambda_{u} \geq 1 / 18$ is reduced to less than $1 / 50$ of that of $\mathrm{HU}$.

Finally, we address the applicability of the scheme presented in this paper. Obviously, it may not work in the hybrid undulator configuration. Together with the fact that the maximum $K$ value is reduced by a factor of $\sqrt{2}$, this scheme may not be desirable for application to short-period undulators. On the other hand, the requirement on the $K$ value is usually moderate for long-period undulators, meaning that the proposed scheme, which can nearly cancel out the strong attractive force, is an attractive option for long-period undulators and will lead to a new concept of undulator structure.

\section{APPENDIX: PCU WITH EIGHT MAGNETS IN A PERIOD}

The Halbach configuration which has four magnets per period $(M=4)$ is commonly used. If we take $M=8$, the magnetic field is enhanced by $8 \%$ according to $\operatorname{sinc}(\pi / 8) /$ $\operatorname{sinc}(\pi / 4)$, which may be an attractive option especially for long-period undulators.

(a)

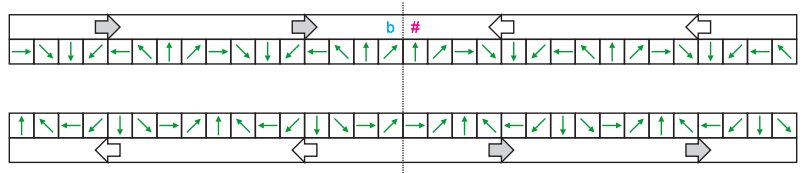

(b)

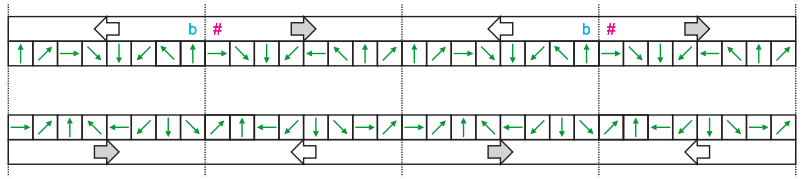

FIG. 9. Conceptual drawing of PCU which has eight magnets per period $(M=8)$ for (a) $D=2$ and (b) $D=N_{u}$. 
Figure 9 shows the conceptual drawing of the PCU which has eight magnets per period $(M=8)$ for (a) $D=2$ and (b) $D=N_{u}$. The relative longitudinal displacement between the upper and the lower magnet arrays is the same as the PCU $(M=4), \lambda_{u} / 4$. Although it is also possible to consider the PCU for $M=2,6,10$, etc., magnets having different thicknesses are required for them. As shown in the figure, the complexities to make the PCU $(M=8)$ are the same as the $\mathrm{HU}(M=8)$.

[1] P. Emma et al., Nat. Photonics 4, 641 (2010).

[2] T. Ishikawa et al., Nat. Photonics 6, 540 (2012).

[3] B. W. J. McNeil, N. R. Thompson, and D. J. Dunning, Phys. Rev. Lett. 110, 134802 (2013).

[4] J. Wu et al., in Proceedings of the 4th International Particle Accelerator Conference, IPAC-2013, Shanghai, China, 2013 (JACoW, 2013), p. 2068.

[5] T. Tanaka, Phys. Rev. Lett. 110, 084801 (2013).

[6] Z. Huang and I. Lindau, Nat. Photonics 6, 505 (2012).
[7] O. Marcouillé, in Proceedings of the International Particle Accelerator Conference, Kyoto, Japan (JACoW, 2010), p. WEPD009.

[8] T. Bizen, in Proceedings of the 8th International Conference on Synchrotron Radiation Instrumentation, San Francisco, 2003 (American Institute of Physics, College Park, USA, 2004), pp. 175-178.

[9] R. Kinjo, T. Seike, A. Kagamihata, S. Yamamoto, and T. Tanaka, in Proceedings of the 36th International Free Electron Laser Conference, Basel, 2014 (JaACoW, 2014), p. MOP023.

[10] S. Yamamoto, J. Phys. Conf. Ser. 425, 032014 (2013).

[11] S. Yamamoto, in Proceedings of the 5th International Particle Accelerator Conference, Dresden, 2014 (JaACoW, 2014), p. WEOAA02.

[12] K. Halbach, Nucl. Instrum. Methods Phys. Res. 187, 109 (1981).

[13] T. Tanaka and H. Kitamura, J. Synchrotron Radiat. 8, 1221 (2001).

[14] K. Halbach, Nucl. Instrum. Methods 169, 1 (1980).

[15] O. Chubar, P. Elleaume, and J. Chavanne, J. Synchrotron Radiat. 5, 481 (1998). 